\title{
La planificación tributaria internacional The international tax planning
}

\author{
Romeo Carpio Rivera* \\ rfcarpio@sri.gob.ec
}

\begin{abstract}
Resumen
La planificación tributaria internacional se ha desarrollado fundamentada en la globalización y tiene su principal asidero en los múltiples beneficios que el propio legislador ha incorporado en la legislación, en su afán de atraer a los países inversión extranjera directa. Existe una gama muy variada de definiciones sobre la planificación tributaria, la elusión y la evasión.
\end{abstract}

\section{Palabras clave}

Actuaciones lícitas, contribuyente, planificación fiscal, elusión, evasión, medida antielusión.

\section{Abstract}

International Tax Planning has been developed based upon globalization and has its main stronghold in the multiple benefits that congressmen has incorporated in their own legislation, with the purpose of attracting direct foreign investment to their countries. There is a wide variety of definitions about tax planning, tax eluding, and tax evasion

\section{Key words}

Legal acting, taxpayer, fiscal planning, elude and tax evasion measure.

Forma sugerida de citar: Carpio, Romeo. 2012. "La planificación tributaria internacional", en: Revista Retos, Año 2, Núm. 3, pp. 53-67. Quito: Editorial Abya Yala.

\footnotetext{
* Maestría Internacional en Tributación y Hacienda Pública, Jefe Nacional de Grandes Contribuyentes.
} 


\section{Introducción}

La planificación tributaria internacional "es un proceso, constituido por una serie de actos o actuaciones lícitas del contribuyente, cuya finalidad es invertir eficientemente los recursos destinados por éste al negocio de que se trata y con la menor carga impositiva que sea legalmente admisible, dentro de las opciones que el ordenamiento jurídico contempla" (Rivas, 2000: 9).

"Toda planificación fiscal persigue minimizar la carga tributaria derivada del ejercicio de una actividad mercantil o de la tenencia de un patrimonio mediante la elección de la vía de acción más eficiente entre todas las alternativas legalmente posibles" (Arepacochaga, 2000: 10).

"Cualquier decisión del obligado tributario que permita minorar sus obligaciones tributarias y que no se oponga, ni siquiera indirectamente, a lo dispuesto en la normativa tributaria" (Ruiz Toledano, 2000: 10).

"Consiste en la facultad de elegir entre varias alternativas lícitas de organización de los negocios o actividades económicas del contribuyente, o de incluso de renunciar a la realización de negocios o actividades, todo con el fin de obtener un ahorro tributario" (Ugalde, 2007: 53).

"El concepto de economía de opción, nace del criterio de que nadie está obligado a elegir el camino más gravoso para el pago de sus impuestos, dada la necesaria libertad para elegir la forma jurídica más conveniente para el cumplimiento de los fines económicos que se persiguen, por tanto cada persona puede arreglar sus asuntos en la forma que le resulte menos onerosa, en consecuencia, se puede elegir la forma jurídica más conveniente para el cumplimiento de los fines económicos que se proponen las partes. este criterio tiene su fundamento en la autonomía de la voluntad, la libertad de empresa, libertad de contratación, libertad económica y en los principios constitucionales y tributarios de reserva de Ley y legalidad." (Yupangui 2010, 8).

\section{Conceptos fundamentales}

\section{Sobre la Elusión Tributaria:}

"La 'elusión tributaria' es toda conducta dolosa del contribuyente que tiene como finalidad evitar el nacimiento de una obligación tributaria, valiéndose para ello de fraude de ley ${ }^{1}$, de abuso de derecho ${ }^{2}$ o de cualquier otro medio ilícito que no constituya infracción o delito" (Rivas, 2000: 21).

\section{Sobre la evasión tributaria:}

"La evasión tributaria es toda conducta ilícita del contribuyente, dolosa o culposa, consistente en un acto o en una omisión, cuya consecuencia es la sustracción al pago de una obligación tributaria que ha nacido válidamente en la vida del derecho, mediante su ocultación a la administración tributaria, en perjuicio del patrimonio estatal" (Rivas, 2000: 29). 
La planificación fiscal versus la elusión y la evasión

$\rightarrow$ Al diferenciar la planificación tributaria y la elusión:

En la elusión existe un escalón o un grado superior a la simple planificación tributaria, puesto que en la primera son muchas las ocasiones en que los contribuyentes utilizan formas jurídicas infrecuentes o atípicas o bien "se aprovecha” de lagunas, vacíos, errores o contradicciones del legislador.

En cambio en la planificación, el contribuyente opta por una de las alternativas dispuestas por el legislador en forma expresa o implícita para que los particulares organicen sus negocios.

Si debemos buscar una similitud entre la economía de opción o planificación tributaria y la elusión debemos concluir que ambas se asemejan a ser actividades lícitas. ${ }^{3}$

èAl diferenciar la evasión y la elusión:

La diferencia está en que en la elusión el contribuyente impide el nacimiento de la obligación tributaria situándose en una zona en donde no llega la ley tributaria mediante un medio lícito, aún cuando sea infrecuente.

En cambio, en la evasión la obligación tributaria nació y el contribuyente mediante una maniobra dolosa impide que la administración tributaria la conozca o permite que la conozca, pero no por su verdadero monto.

Así, por ejemplo, el contribuyente impide que la administración conozca la existencia de la obligación tributaria cuando maliciosamente omite la declaración impositiva. En cambio, el contribuyente impide que la administración conozca el verdadero monto de la obligación cuando maliciosamente incorpora facturas falsas a su contabilidad, a fin de rebajar gastos que, en definitiva, resultan ser inexistentes.

La Elusión Tributaria se diferencia de la evasión tributaria porque el uso de ciertos tecnicismos legales le otorga absoluta legitimidad, para efectos jurídicos. No obstante, se discute sobre la legalidad de dichos actos, puesto que, en la mayoría de los casos, el aprovechamiento de subterfugios legales se lo hace en forma maliciosa y con miras a no pagar tributos o pagarlos en forma disminuida, perjudicando en esta forma al estado, por ello, hay quienes afirman que estas conductas destinadas a rebajarse los impuestos pueden ser consideradas delictivas, si la propia Ley Tributaria consigue tipificar a la Elusión Tributaria y le imponga las sanciones que castiguen esta conducta como hecho punible (Yupangui, 2010: 3).

\section{$\rightarrow \quad$ Al diferenciar la planifica-}

ción, la evasión y la elusión:

En la economía de opción (planificación tributaria), el contribuyente sabe que está actuando dentro de las posibilidades que la Ley le brinda y la pasividad del intérprete le ratifica que la norma no ha querido gravar el resultado alcanzado.

En la elusión, el contribuyente cree que está alcanzando un ahorro fiscal legítimo, pero su conducta es 
recalificada fiscalmente, porque produce una tensión con el propósito atribuido a la ley fiscal, que se propuso alcanzar esa situación.

En la evasión, el contribuyente sabe que está quebrando la ley, no evita el hecho imponible, sino que solo intenta sustraerse a la obligación tributaria mediante ocultaciones maliciosas o declaraciones engañosas que pueden hallar soporte funcional en el empleo de formas jurídicas verdaderamente inadecuadas que se exhiben como mera pantalla para ocultar la realidad.

Dado que en algunas acciones puede ser muy tenue la diferencia, sobre todo entre la planificación y elusión, propongo hacer una diferenciación a partir de la legitimidad de las actuaciones; tanto desde un punto de vista legal, cuanto filosófico; entendiendo la primera como una legitimidad que se evidencia cuando la acción se enmarca dentro de las normas legales y la segunda a aquella que cuenta con el reconocimiento social; como se muestra en el siguiente cuadro:

CUADRO No 1

\begin{tabular}{|l|c|c|}
\cline { 2 - 3 } \multicolumn{1}{c|}{} & \multicolumn{2}{c|}{ LEGITIMIDAD } \\
\cline { 2 - 3 } \multicolumn{1}{c|}{} & LEGAL & FILOSOFICA \\
\cline { 2 - 3 } \multicolumn{1}{c|}{} & & (RECONOCIMIENTO \\
\hline PLANIFICACION & SI & SI \\
\hline ELUSION & SI & NO \\
\hline EVASION & NO & NO \\
\hline
\end{tabular}

ELABORACIÓN: EL AUTOR
Lo que es legítimo desde un punto de vista jurídico, no necesariamente lo es desde un punto de vista filosófico o social. Por ejemplo, podemos traer a colación el clásico cuento de Robin Hood, quien robaba a los ricos y poderosos para dárselos a los pobres.

Desde un punto de vista puramente legal se concluiría que sus actuaciones eran ilegales y expuestas a las sanciones correspondientes, pero para la percepción de la sociedad (cuando la mayoría de aquella era pobre) estas actuaciones podrían ser vistas como válidas y envestidas con un carácter de legítimas.

En este sentido, es interesante lo manifestado por el profesor Gerard Verna (1993) en su trabajo Cuando lo legal no es bueno y lo ilegal no es malo, en donde analiza las acciones desde la discrepancia que a su juicio existe entre la legalidad y la legitimidad, describiéndolas como dos nociones distintas.

En ese trabajo, el profesor Verna empieza su análisis categorizando a las acciones en cuatro tipos, dependiendo del grado de legalidad o legitimidad que cada una tiene:

- Acciones normales

- Acciones de violencia legal

- Acciones criminales

- Acciones informales

Se identifican claramente a través del siguiente gráfico: 
GRAFICO No1

Categorización de las acciones

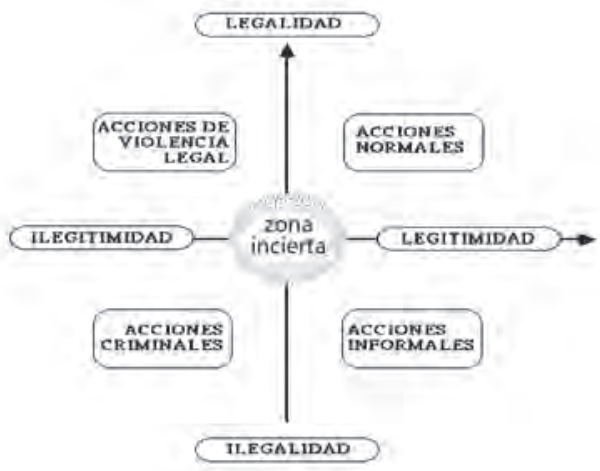

FUENTE: Gérard Verna

Basado en este modelo, se plan- vista legal, cuanto desde lo filosófico; tea diferenciar las actuaciones rela- relacionado con la norma y con el recionadas al ámbito fiscal, conside- conocimiento social, respectivamenrando la legitimidad de las acciones te. Para lo cual, se plantea el siguiende evasión, tanto desde un punto de te gráfico:

Grafico N. 2

Los sentidos de la legitimidad

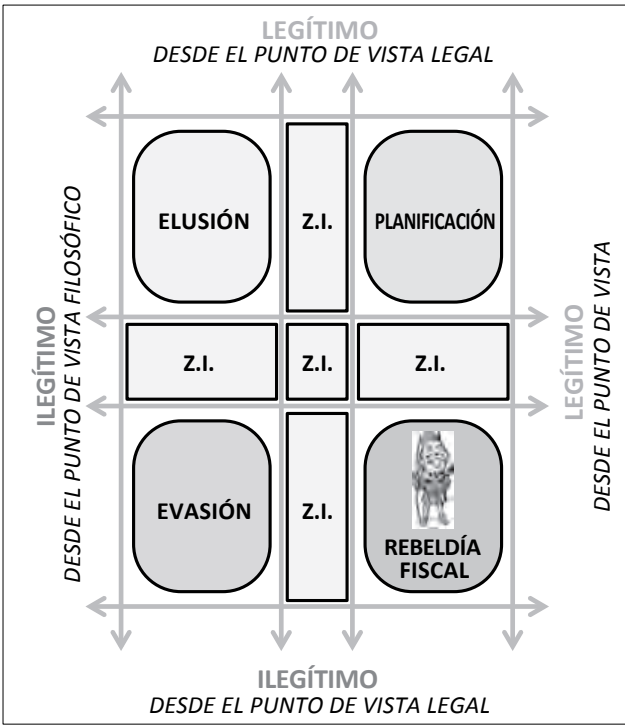

ELABORACIÓN: EL AUTOR 
Existe un cuadro central conformado por tres filas y por tres columnas, las filas tienen relación con la legitimidad desde el punto de vista legal y las columnas con la legitimidad desde lo filosófico.

$\mathrm{Al}$ analizar las filas podría entenderse que las actuaciones deberían ser concebidas como legales (arriba) o ilegales (abajo), con ello se platearía que solo existirían dos alternativas; pero teniendo en cuenta que la interpretación jurídica, contiene un cierto grado de subjetividad, entonces surge la posibilidad de que una actuación, en un punto determinado, no pueda ser claramente identificable desde una mirada de lo legalmente aceptable, dado lugar a una "zona incierta" (lo que en el gráfico se observa como Z.I.).

Algo similar sucede con las columnas, las actuaciones tienen o no reconocimiento social podrían o no ser legítimas desde ese punto de vista. En ese caso también existirían dos alternativas; pero teniendo en cuenta que la aceptación social depende de la idiosincrasia de los individuos, su cultura, costumbres, religión, etcétera surge también la posibilidad de que una actuación no cuente claramente con el reconocimiento social y pueda ser clasificada dentro de una "zona incierta".

Entonces, es fácil comprender lo planteado en el cuadro No. 1, donde se estableció una gran diferencia entre la planificación y la elusión, con la evasión; pues las primeras se las concibe como legítimas desde los sentidos de lo legal, y la última es transgresora. Se entiende que las actuaciones que transgreden la Ley, no pueden ser vistas como planificación ni como elusión fiscal.

Tanto la planificación como la elusión son legales y; por ende, legítimas desde ese punto de vista, pero no sucede lo mismo desde la perspectiva filosófica donde la planificación es aceptada y reconocida por la sociedad como legítima, pero la elusión tributaria no; y menos aún la evasión.

En el gráfico No. 2 las actuaciones legítimas se las denomina planificación tributaria, (legítimas desde un punto de vista legal pero no filosófico), desde lo justificado se las conoce como elusión tributaria y que aquellas que no poseen ningún grado de legalidad son concebidas como evasión. El último cuadrante pertenece para aquellas acciones que a pesar de ser ilegales cuentan con un reconocimiento social, similar a lo que sucedía con Robin Hood; pero en el ámbito fiscal, son entendidas como rebeldía fiscal y no cuentan con apoyo y reconocimiento de la sociedad.

De regreso a los tres conceptos que interesan en este análisis, el modelo se complica cuando hay actuaciones que caen dentro de las zonas de incertidumbre, porque no son fácilmente identificables como legítimas o ilegítimas, entonces en esos casos los conceptos pueden llegar a ser confundidos como se los muestra en el siguiente gráfico: 
Gráfico N. 3

Lo legítimo e ilegítimo de elusión, planificación, evasión, rebeldía

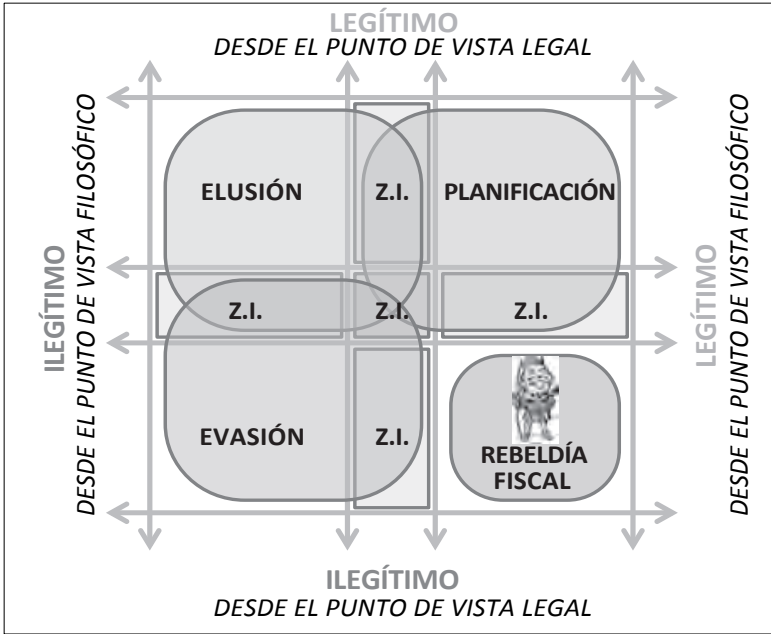

ELABORACIÓN: EL AUTOR

Un caso práctico, aplicado a la legislación ecuatoriana, las empresas $\mathrm{A}, \mathrm{B}$ y $\mathrm{C}$ son empresas residentes en Ecuador y en su afán de aminorar la carga fiscal han decidido contratar sendos asesores tributarios para que realicen la planificación fiscal del año 2011 y la consecuente obtención de la máxima utilidad para sus accionistas.

Una vez concluidos los trabajos de los asesores se observa que dentro de las planificaciones propuestas y específicamente en la parte que trata sobre la contratación de personal se evidencian las siguientes formaciones:

EMPRESA A: "En relación con la contratación de personal, se ha identificado que está previsto adjuntar diez nuevos empleados en el mes de agosto para las labores de oficiales de cuenta, con una remuneración mensual de USD 2.000 dólares cada uno; y en vista de que la legislación vigente prevé un beneficio tributario para la contratación de personal que sea parte de la compañía al menos por seis meses. Se propone que las contrataciones se adelanten al primer día de julio, esto generaría, en términos netos, un importante beneficio para la compañía."

EMPRESA B": "En relación con la contratación de personal, se ha identificado que no está previsto contratar nuevos empleados en el siguiente año, pero también se tiene en consideración que existe la compañía $\mathrm{X}$, que es parte relacionada de la compañía B; y en vista de que la legislación vigente prevé un beneficio tributario para la contratación de personal que sea parte de la 
compañía al menos por seis meses, se propone que se identifiquen los empleados a los que se le podría proponer que cambien su relación laboral de la compañía X a la compañía B a pesar de que continúen trabajando para la compañía X (sería únicamente una variación en las nóminas y contratos de cada empresa), una vez definido, se realice sus traslados en el mes de enero para lograr obtener el mayor beneficio posible, pues esto generaría, en términos netos, un importante beneficio para la compañía."

EMPRESA C: "En relación con la contratación de personal, se ha identificado que no está previsto contratar nuevos empleados en el siguiente año; pero considerando que en la legislación vigente se prevé un beneficio tributario para la contratación de personal que sea parte de la compañía al menos por seis meses, se propone incorporar dentro de la nómina personas mediante su contratación ficticia, para lo cual se puede utilizar personal que se encuentran fuera del país y que se prevé no volverán al mismo en el largo plazo. A más del beneficio adicional que otorga la Ley, evidentemente se reducirá la base imponible por los sueldos y salarios que se registren a sus nombres."

Seguramente es sencillo definir dentro de que categoría se puede clasificar a cada una de las anteriores propuestas, en el primer caso, al aprovechar de un beneficio/incentivo planteado por el legislador, estaríamos claramente en un caso de planificación; mientras que en el segundo, se incorpora una actuación del contribuyente que va en contra del incentivo del legislador; es decir, el incremento de empleo neto; el propio legislador en nuestro cuerpo legal, incorporó una medida antielusión que precisamente evita esta posibilidad; y en el caso de la empresa C, al inventar, maliciosamente, gastos que resultan ser inexistentes, se trata de un caso de evasión.

Para entender mejor el cuadro planteado anteriormente, donde se diferencia estos conceptos desde una perspectiva de legitimidad legal y de legitimidad filosófica, lo podemos aplicar al ejemplo anterior:

- En el caso A, se cumple la norma legal y existiría aceptación social, pues realmente se genera el objetivo de la norma, que es el crecimiento del empleo neto.

- En el caso B (manteniendo el supuesto de que la norma no incluya la medida antielusión), se cumple la norma legal pero no el objetivo de la norma, se podría presumir que no existiría una verdadera aceptación social y por ende carecería de legitimidad social.

- Por último, en el caso C, no existe cumplimiento de las normas vigentes y menos aún existiría aceptación social, tanto legitimidad legal cuanto social no cabrían en este caso. 
CUADRO No 2

\begin{tabular}{|l|c|c||}
\cline { 2 - 3 } \multicolumn{1}{c|}{} & \multicolumn{2}{c|}{ LEGITIMIDAD } \\
\cline { 2 - 3 } \multicolumn{1}{c|}{} & LEGAL & FILOSOF. \\
\hline CASO “A" & SI & SI \\
\hline CASO "B" & SI & NO \\
\hline CASO "C" & NO & NO ELNIFICACIÓN \\
\hline
\end{tabular}

\section{La planificación fiscal internacional}

Para muchos autores, teniendo en cuenta el tipo de transacciones que son objeto de planificación y si están relacionadas dentro del mercado local o no, surge una clasificación que diferencia a la planificación fiscal local de la planificación fiscal internacional (PFI), siendo esta última la que en este análisis toma mayor relevancia.

"Cuando se habla de planificación fiscal internacional, muchas veces se piensa en la pura elusión, cuando no evasión o fraude fiscal, que las empresas multinacionales o transnacionales llevarían a cabo aprovechando su presencia en múltiples jurisdicciones o países. En realidad, la verdadera planificación fiscal internacional es el resultado de la existencia de una multiplicidad de regímenes fiscales nacionales que ofrecen así a estas empresas con una presencia internacional la opción o la posibilidad de tomar decisiones empresariales atendiendo entre otros factores al régimen fiscal aplicable.

No puede hablarse de planificación fiscal cuando las empresas simplemente ocultan o alteran la realidad o trascendencia de sus operaciones con propósitos de fraude o evasión fiscal" (De la Cueva, 2008: $5)$.

Pero, ¿basada en qué surge la PFI? Sin lugar a duda esta surge básicamente en los siguientes aspectos:

El crecimiento acelerado del comercio internacional como parte del proceso de globalización. "El problema que de forma más clara e intensa ha influido en las estructuras económicas, sociales y políticas de finales del siglo XX e inicios del siglo XXI ha sido el de la globalización económica. Este fenómeno que se define como la creciente interdependencia económica entre todos los países, provocada por el aumento del flujo de bienes y servicios, de capitales y por la difusión de la tecnología y de la información, ha generado un contexto internacional de interacción entre las economías y una desaparición virtual de las fronteras" (Vallejo, 2003: 499).

La conformación de los grupos multinacionales. "En el contexto de esta evolución de la estructura económica internacional, destaca a nuestros efectos la evolución experimentada por la organización de las 
empresas multinacionales o transnacionales anglosajonas, especialmente las basadas en los Estados Unidos. Estas compañías de base norteamericana han sido y son las que en mayor medida se han internacionalizado, pasando de estructuras internacionales, de base exportadora, a estructuras multinacionales o verdaderamente transnacionales. No obstante, ejemplos de compañías transnacionales podemos encontrar hoy también a nivel europeo en la medida en la cual la integración económica europea va permitiendo la aparición de compañías que realmente no tienen una base nacional exclusiva sino que han resultado de la fusión o combinación de empresas con orígenes nacionales diferentes" (De la Cueva, 2008: 10).

La necesidad de los estados de atraer a la inversión extranjera directa (IED). "En consecuencia, los Estados son en realidad los primeros protagonistas de la verdadera planificación fiscal internacional. Son los Estados los que frecuentemente conducen su política fiscal tratando de hacer que sus regímenes impositivos aparezcan como un atractivo más para la inversión extranjera. Como ya hemos apuntado, es básicamente esa diversidad de regímenes fiscales, incluyendo las redes de convenios de doble imposición de cada país, la que hace que surja la planificación fiscal de las empresas" (De la Cueva, 2008: 12)

El bajo costo de implementar normas que atraigan las decisiones de los grupos multinacionales. Cuando el legislador analiza la incorporación de un incentivo fiscal, normalmente la contraparte es el coste en la recaudación; por ejemplo, tomando nuevamente el ejemplo de la norma que busca el crecimiento del empleo neto, el costo será la reducción de recaudación en el impuesto a la renta que esto generaría, y en consecuencia, si para obtener un beneficio extrafiscal, el costo relacionado con el es asimilable en términos relativos con el beneficio que se obtiene, entonces se considera prudente su incorporación; es así como en cada ocasión que el legislador analiza la posibilidad de incorporar un nuevo incentivo o beneficio debe realizar análisis de costo beneficio. Pero, ¿qué pasa cuando el incorporar una norma que genera un beneficio extrafiscal no tiene ningún costo en la recaudación? Normalmente lo que sucede cuando un Estado desea incorporar normas con la finalidad de atraer IED, pues su beneficio extrafiscal es alto, mientras que su costo fiscal suele ser nulo, ya que de no existir la norma propuesta, la inversión no existiría en el país. En ese sentido el límite para los Estados está en la aceptación por parte de la comunidad internacional, de no definir tales medidas como perniciosas. Un ejemplo interesante de analizar es el caso Irlandés, en donde con el propósito de atraer IED se llegó a reducir la tarifa general del impuesto a la renta a niveles del 10\%, pero ante el reclamo de la comunidad inter- 
nacional, esta fue elevada al 12,5\%, conjuntamente con otras medidas que permitan tranquilizar a los demás países de la Comunidad Europea.

Normalmente la PFI es analizada desde cinco opciones básicas:

1. Ubicación de las actividades productivas.- Al momento de tomar una decisión respecto de la ubicación de las actividades productivas, son muchos los factores que deben tenerse en cuenta, y normalmente el fiscal no suele ser el más importante; entre otros, tenemos la seguridad jurídica, los regímenes laborales y legales aplicable a inversiones extranjeras, riesgo cambiario, administración pública local, sistema económico, factores sociales, lingüísticos, religiosos, subvenciones y ayudas e incluso el régimen aduanero.

2. Estructura y centros financieros y de servicios.- "Uno de los terrenos donde más frecuentemente actúan los esquemas de planificación fiscal internacional es el de las rentas asociadas a la financiación de los grupos de sociedades. Las técnicas o esquemas de planificación en relación con los centros financieros o de servicios, suponen la búsqueda de unos gastos deducibles que representan unos ingresos computables en una jurisdicción distinta, siendo menor la tributación aplicable sobre esas rentas en esta segunda jurisdicción" (De la Cueva, 2008: 46).

3. Intangibles.- Al momento de valorar una empresa, toma importancia el valor de sus intangibles, donde se pueden encontrar muchos conceptos como el propio fondo de comercio o el valor de la misma organización empresarial, y una serie de derechos valorables económicamente, sean o no susceptibles de registro o protección legal como tales, que han resultado de la propia vida de la empresa o que ha comprado directamente a terceros. Si bien a los intangibles se los puede clasificar sobre la base de distintos criterios, en este análisis vamos a tomar en cuenta una clasificación sencilla que los diferencia entre industriales y comerciales.

4. Estructuras corporativas y sociedades holding.- "Básicamente, estas sociedades pueden ser sociedades cabeceras de un grupo, admitidas inclusive a cotización; pueden ser operativas al mismo tiempo que tenedoras de las participaciones; o pueden ser puras sociedades holding limitadas en su papel a ser tenedoras de las participaciones de las filiales o subsidiarias del grupo en un determinado sector económico o área geográfica. Desde la perspectiva de la planificación fiscal internacional, son estas últimas entidades, estas sociedades subholding las que nos interesan 
especialmente y las que reciben en muchas jurisdicciones un tratamiento fiscal específico" (De la Cueva, 2008: 60)

5. La política de precios de transferencia.- Como se ha manifestado en varias ocasiones, la PFI se fundamenta generalmente en el desarrollo de los grupos económicos transnacionales, y en sentido tiene mucha importancia la valoración de las transacciones intragrupo. Bajo un esquema puro de planificación tributaria, en el ámbito de los precios de transferencia, las empresas procuran definir precios que minimicen la posibilidad de ser objetados por las administraciones tributarias, generándose los supuestos indeseables de doble imposición.

\section{Los centros financieros}

Cuando los grupos económicos se desarrollan, de los excedentes que sus empresas generan y otras de las necesidades de financiamiento, entonces surge la necesidad de crear centros financieros destinados exclusivamente a atender las necesidades propias del grupo, o también, a atender las de terceros.

\section{Los paraísos fiscales}

Se entiende normalmente que un paraíso fiscal es un país o una jurisdicción que ofrece condiciones favorables en términos fiscales para los no residentes que deciden invertir en su territorio.

Por la diversidad que plantean, no es sencillo establecer una defini- ción, la Organización para la Cooperación y el Desarrollo Económico OCDE (1998), han preferido establecer los rasgos característicos que perfilan a lo que puede considerarse como un paraíso fiscal, principalmente:

\section{Medidas antiparaísos}

Hoy en día, la utilización de paraísos fiscales no forma parte importante de la planificación fiscal de los grupos multinacionales, básicamente debido a las medidas antiparaísos que han adoptado los propio países y por otro lado, razones de imagen que hacen delicada la presencia de inversiones o de actividades, un ejemplo es toda la información que en el mundo recorrió los últimos meses del año anterior en referencia con los ahorros tributarios generados por Google a través de un esquema de planificación fiscal que ha utilizado empresas ubicadas en Irlanda, Holanda y Bermuda.

Google rebajó sus impuestos en unos 3.100 millones de dólares (unos 2.200 millones de euros) durante los últimos tres años usando técnicas que transfieren la mayor parte de sus beneficios de fuera de Estados Unidos a las islas Bermudas a través de Irlanda y Holanda.

Google no ha sido acusada de vulnerar las normas fiscales. "Las prácticas de Google son muy similares a las de muchas otras firmas globales de varios sectores”, según un portavoz de la empresa que declinó 
dar detalles sobre su estrategia fiscal (El País, 2010).

\section{El caso ecuatoriano}

Al revisar el texto de la Ley de Régimen Tributario Interno que se encontraba vigente hasta el año 2007, curiosamente se puede apreciar que en ningún artículo se hace mención o se restringe la utilización de paraísos fiscales, recientemente con la LET y posteriores reformas, se incorporan en la Ley algunas medidas relacionadas con paraísos fiscales.

1. Se considera que se han efectuado transacciones con partes relacionadas cuando estas sociedades se encuentren ubicadas, constituidas o domiciliadas en paraísos fiscales.

2. La ley otorga a la administración tributaria la facultad de determinar, mediante resolución, los países y las jurisdicciones que sean consideradas como paraísos fiscales, para fines tributarios.

3. Exoneración del impuesto a la renta en dividendos distribuidos por sociedades nacionales a sociedades extranjeras, excepto en el caso que estas se encuentren domiciliadas en paraísos fiscales.

4. Reducción del porcentaje de retención de impuesto a la renta por pagos efectuados al exterior por concepto de intereses, cuando el beneficiario de estos no se encuentre domiciliado en un paraíso fiscal.

5. No deducibilidad de los cánones o cuotas pagados por concepto de arrendamiento mercantil internacional, cuando el beneficiario de los mismos se encuentre domiciliado en un paraíso fiscal.

6. Condición dentro del puerto seguro de precios de transferencia, de que el contribuyente que se acoja al puerto seguro no haya efectuado ninguna operación con residentes en paraísos fiscales.

7. Exoneración de las rentas provenientes del extranjero, cuando estas no hayan sido obtenidas en paraísos fiscales.

El Código Orgánico de la Producción, Comercio e Inversiones, en su artículo 14 dispone que los beneficios establecidos en el Código, no se aplicarán a aquellas inversiones de personas naturales o jurídicas extranjeras domiciliadas en paraísos fiscales. Con lo cual, incluso la reducción de la tarifa de impuesto a la renta de sociedades, no aplicaría en los casos definidos en el artículo mencionado.

También existen otras normas establecidas mediante reglamento, como las siguientes:

1. No se acepta la exoneración sobre la retención de impuesto en el caso de pagos por concepto de comisiones en exportaciones, cuando el beneficiario de las mismas resida en un paraíso fiscal.

2. Igual que en el caso anterior, sucede con las comisiones pagadas por promoción del turismo receptivo. 


\section{Conclusiones y recomendaciones}

- Existe una gama muy variada de definiciones sobre la planificación tributaria, la elusión y la evasión. Parecería ser que la mayoría coincide en que únicamente las dos primeras son actuaciones lícitas.

- A pesar de que existe una línea muy tenue entre la planificación y la elusión, la manera más adecuada de enmarcar una acción dentro de estas definiciones estaría basada en el reconocimiento social que pueda tener.

- La PFI se ha desarrollado fundamentada en la globalización, y tiene su principal asidero en los múltiples beneficios que el propio legislador ha incorporado en su propia legislación, en su afán de atraer a los países inversión extranjera directa.

- Normalmente la PFI es analizada desde cinco opciones básicas: Ubicación de las actividades productivas, estructura, centros financieros y de servicios, intangibles, estructuras corporativas y sociedades holding; y la política de precios de transferencia.

- En el caso ecuatoriano debería analizarse la conveniencia de incorporar un régimen tributario favorable para incentivar el traslado de expatriados al país, teniendo en consideración que aportan a la generación de conocimiento.
- En el crecimiento de los grupos multinacionales, aparecen empresas deficitarias de recursos financieros y otras excedentarias, con lo cual surge la necesidad de crear centros financieros, con el fin de que estos grupos tomen las decisiones adecuada en cuanto a su economía.

- En el caso ecuatoriano se incorporó una norma especial para controlar la subcapitalización, hasta entonces la administración tributaria contaba únicamente con una norma antielusión general. No se puede afirmar que a partir del año 2008 la norma de subcapitalización aplica como un verdadero "puerto seguro" para el endeudamiento con partes relacionadas en el extranjero, en caso de que una transacción carezca de esencia económica, el pago de intereses y toda la operación deben ser desconocidos y en tal medida, no cabe la norma particular.

- Figuras de supuesta planificación fiscal internacional que carecen de esencia económica no pueden ser concebidas como planificación, sino elusión o evasión.

- En Ecuador, las normas anti paraísos fiscales solo aparecen a partir del año 2008 y en términos relativos son aún muy escasas y leves; por lo tanto es recomendable que se incluya mayor regulación contra las operaciones efectuadas con la intervención de 
paraísos fiscales que carecen de sustancia económica. Dentro de esto se debería también restringir el cambio de domicilio fiscal de las personas naturales a paraísos fiscales.

\section{Notas}

1 Según el propio autor, fraude de ley es una especie de elusión mediante la cual el contribuyente evita, aparentemente, el nacimiento de la obligación tributaria, amparándose en una norma no adecuada a la actividad que desarrolla con el fin de defraudar otra que si corresponde a dicha actividad o supuesto de hecho.

2 Según el propio autor, abuso de derecho en materia tributaria, es una especie de elusión que se configura cuando el contribuyente extralimita el poder que se le ha conferido por una norma jurídica, con el fin de evitar el nacimiento de una obligación tributaria o de reducir su monto, en perjuicio del Fisco.

3 Conforme a la Real Academia Española, lícito: que es de la ley o calidad debida.

4 Para facilitar el entendimiento se incorpora un supuesto, de que en la legislación ecuatoriana la norma no prevé la prohibición de que los empleados hayan pertenecido anteriormente a empresas que sean relacionadas.

\section{Bibliografía}

CÓDIGO ORGÁNICO TRIBUTARIO

CÓDIGO ORGÁNICO DE LA PRODUCCIÓN, COMERCIO E INVERSIONES

DE LA CUEVA González

COTERA, Álvaro.

(2008) La Planificación Fiscal Internacional, Madrid: Instituto de Estudios Fiscales.
GOMEZ, Acebo

SOLAR, Ricardo

(2008) Medidas Antielusión. Marco Fiscal de la Unión Europea. Nuevos Problemas y Tendencias en Fiscalidad Internacional. Madrid: Instituto de Estudios Fiscales.

LEY DE RÉGIMEN TRIBUTARIO INTERNO

LOPEZ Ribas, Silvia

(2008) Figuras Impositivas: Impuesto sobre la Renta. Madrid: CEDDET

OCDE

(1998) Harmful Tax Competition, an emerging global issue.

RIVAS CORONADO, Norberto

(2000) Planificación Tributaria, Conceptos, Teoría y Factores a Considerar.

Santiago de Chile: Editorial Magril Limitada. Texto Refundido del IRS Español.

GARCIA Escobar, Jaime

(2007) Elusión, Planificación y Evasión Tributaria. Santiago de Chile: Lexis Nexis.

VALLEJO, José María.

(2003) "La Competencia Fiscal Perjudicial", en: Fiscalidad y Planificación Fiscal Internacional. Madrid: Instituto de Estudios Económicos.

YUPANGUI CARRILLO, Yolanda.

(2010) Posibilidad de Evitar la Elusión Tributaria y Figuras Relacionadas.

Ponencia presentada en las XXV Jornadas Latinoamericanas de Derecho Tributario. Cartagena-Colombia, febrero.

El país

(2010) "Google usa paraísos fiscales para pagar solo un $2,4 \%$ de impuestos". Tomado de: http://elpais.com/diario/2010/10/22/ economia/1287698406_850215.html.

Fecha de recepción: 10/11/2011. Fecha de aceptación: 16/05/2012 